\title{
Structure of entangled polymer network from primitive chain network simulations
}

\section{AUTHOR(S):}

Masubuchi, Yuichi; Uneyama, Takashi; Watanabe, Hiroshi; Ianniruberto, Giovanni; Greco, Francesco; Marrucci, Giuseppe

\section{CITATION:}

Masubuchi, Yuichi ...[et al]. Structure of entangled polymer network from primitive chain network simulations. JOURNAL OF CHEMICAL PHYSICS 2010, 132(13): 134902.

\section{ISSUE DATE:}

2010-04

URL:

http://hdl.handle.net/2433/147217

\section{RIGHT:}

Copyright 2010 American Institute of Physics. This article may be downloaded for personal use only. Any other use requires prior permission of the author and the American Institute of Physics. The following article appeared in JOURNAL OF CHEMICAL PHYSICS132, 134902 (2010) and may be found at 


\title{
Structure of entangled polymer network from primitive chain network simulations
}

\author{
Yuichi Masubuchi, ${ }_{4}^{1,2, a)}$ Takashi Uneyama, ${ }^{1,2}$ Hiroshi Watanabe, ${ }^{1}$ Giovanni lanniruberto, ${ }^{3}$ \\ Francesco Greco, ${ }^{4}$ and Giuseppe Marrucci ${ }^{3}$ \\ ${ }_{1}^{1}$ Institute for Chemical Research, Kyoto University, Gokasyo, Uji, Kyoto 611-0011, Japan \\ ${ }^{2}$ JST-CREST, Kyoto 611-0011, Japan \\ ${ }^{3}$ Dipartimento di Ingegneria Chimica, Università degli studi di Napoli “Federico II," Piazzale Tecchio, \\ 80-80125 Napoli, Italy \\ ${ }^{4}$ Istituto di Ricerche sulla Combustione, CNR, Piazzale Tecchio, 80-80125 Napoli, Italy
}

(Received 15 January 2010; accepted 3 March 2010; published online 1 April 2010)

\begin{abstract}
The primitive chain network (PCN) model successfully employed to simulate the rheology of entangled polymers is here tested versus less coarse-grained (lattice or atomistic) models for what concerns the structure of the network at equilibrium (i.e., in the absence of flow). By network structure, we mean the distributions of some relevant quantities such as subchain length in space or in monomer number. Indeed, lattice and atomistic simulations are obviously more accurate, but are also more difficult to use in nonequilibrium flow situations, especially for long entangled polymers. Conversely, the coarse-grained PCN model that deals more easily with rheology lacks, strictly speaking, a rigorous foundation. It is therefore important to verify whether or not the equilibrium structure of the network predicted by the PCN model is consistent with the results recently obtained by using lattice and atomistic simulations. In this work, we focus on single chain properties of the entangled network. Considering the significant differences in modeling the polymer molecules, the results here obtained appear encouraging, thus providing a more solid foundation to Brownian simulations based on the PCN model. Comparison with the existing theories also proves favorable. (c) 2010 American Institute of Physics. [doi:10.1063/1.3370346]
\end{abstract}

\section{INTRODUCTION}

It is well known that the dynamics of long chain polymers in concentrated solutions and melts can be described by entanglement-based theories. ${ }^{1}$ Indeed, semiphenomenological theories that represent entanglements, either through tube constraints or via sliplink models, have achieved significant success in predicting the rheology of polymeric liquids, also for complex molecular architectures. ${ }^{2}$ In spite of their success, these theories obviously cannot provide a microscopic description of the entanglement.

Recently however Monte Carlo (MC) and molecular dynamics (MD) simulations have been used to investigate topological constraints among polymer chains. In the pioneering work of Everaers et $a l^{3}$ based on bead-spring chain molecular dynamics, the topology is extracted through a procedure whereby first all chain ends are fixed in space, then the intrachain excluded volume interaction is switched off, and the temperature is progressively reduced to zero, thus making all chains taut. From the obtained topological network, the average molecular weight of the network strands is calculated and shown to be consistent with the entanglement molecular weight experimentally obtained from the plateau modulus. ${ }^{4}$ Subsequently, different techniques have been developed to extract the topology of the entangled network from the minimization of the contour length (i.e., to realize

\footnotetext{
${ }^{\text {a) }}$ Author to whom correspondence should be addressed. Electronic mail: mas@scl.kyoto-u.ac.jp. Tel.: +81-774-38-3136. FAX +81-774-38-3139.
}

the shortest path network), ${ }^{5-12}$ by using either atomistic models ${ }^{5-7,9-12}$ or the bond fluctuation lattice model.

Once the topological structure of the entangled network is determined, the statistics of such structure can be analyzed and compared with existing theories. The classical theory ${ }^{13,14}$ predicts a simple exponential distribution for the number of monomers between neighboring entanglements, whereas a rather complex distribution for the number of entanglements per chain is obtained in Ref. 15. More recent results are as follows. Schieber ${ }^{16}$ theoretically derived the statistics of the topological network based on an assumed free energy for a sliplink model of entangled polymers where (i) entanglements are randomly distributed along the chain, (ii) each subchain between entanglements is assumed to be Gaussian, and (iii) a phenomenological chemical potential is introduced to stabilize sliplinks on the chain. He reported the statistics of subchains in terms of both length and monomer number distribution, the latter being consistent with the classical theory (i.e., exponential). He also calculated that the distribution of the number of entanglements per chain is Poissonian. Subsequent rheological predictions from his sliplink model are shown to be in agreement with experiments. ${ }^{17}$

The simulations of Tzoumanekas and Theodorou ${ }^{7}$ and later studies ${ }^{10,11}$ revealed however that the subchain monomer number distribution is not a simple exponential and shows a maximum at a value somewhat smaller than the mean monomer number. Since the simulation results could be fit by the difference of two negative exponentials, Tzoumanekas and Theodorou ${ }^{7}$ argued that the entanglements are 
not randomly located along the chain. Rather, after each entanglement, there exists a "blocked" region of random length beyond which a new entanglement distance can be randomly chosen. For what concerns the distribution of entanglement number per chain, they pointed out that a Gaussian distribution should be asymptotically approached for long chains and that their simulations do not yet reach the asymptotic behavior.

Finally, Greco ${ }^{18}$ pointed out that the standard statistical treatment for a subchain is not adequate since the number of Kuhn steps in each subchain is too small (in melts even much less than 100). He constructed a single subchain model, where the subchain is connected to a monomer bath at both ends, and the number of monomers is then controlled by a chemical potential. The resulting distribution function of monomer number shows naturally a peak, while the simple exponential distribution is only recovered when the average monomer number goes to infinity. Greco's ${ }^{18}$ distribution is close to (though analytically different from) the one suggested by Tzoumanekas and Theodorou ${ }^{7}$ and is thus consistent with their simulation results. The subchain length distribution, also derived by Greco, ${ }^{18}$ coincides with that of Schieber. $^{16}$

In this study, the network structure obtained by using our primitive chain network $(\mathrm{PCN})$ model $^{19}$ is compared with the above recalled recent results from $\mathrm{MD} / \mathrm{MC}$ simulations and theories, dealing with equilibrium conditions. The PCN model is a multichain sliplink model, which naturally incorporates the known chain dynamical mechanisms (reptation, contour length fluctuations, and constraint release), and additional relaxation mechanisms in the three-dimensional (3D) network (due to force balance on entanglements and to osmotic forces generated by density fluctuations). Simulations based on the PCN model have been found to reproduce linear and nonlinear rheology of entangled polymers quantitatively. $^{20-26}$ Since rheology as considered so far is linked to average network properties only, to confirm the reliability of the coarse-grained PCN model (also accounting for interchain correlations in 3D) it seems appropriate to compare the equilibrium distributions with those obtained from more "microscopic" simulations. In this study, by "equilibrium distributions" we mean those obtained from simulations in the absence of flow, and when any time dependence induced by the initial condition of the simulation box is extinguished.

\section{MODEL AND SIMULATIONS}

\section{A. Sliplink motion and monomer sliding}

In the PCN model, the dynamics of the network formed by the primitive chains is considered. The network nodes stand for entanglements between chains, and at a node two chains are linked by a sliplink, which allows for monomer transport along both primitive chains (i.e., for longitudinal polymer sliding). Chain-end processes account for sliplink removal or creation, thus introducing topological changes in the entanglement network.

In this study, three versions of PCN simulation are examined that differ in the procedure of creation and destruc- tion of sliplinks. These differences may affect the network structure and the statistical properties. Although the PCN model has been described in previous publications, ${ }^{19-26}$ a brief description of the model will be repeated here to better clarify differences and similarities among the three codes.

Hereafter, we choose the average length $a$ of the network strands (i.e., the average distance between consecutive entanglements) as our unit of length; our unit of energy is $k T$, and the unit of time is $\tau=\zeta a^{2} / 6 k T$, where $\zeta$ is the friction coefficient for the motion in space of a sliplink. We also normalize the monomer number $n$ in a subchain to its mean value at equilibrium, $n_{0}$. In the equations shown below, the variables are nondimensional and normalized according to the above.

The equation of motion for the sliplink position $\mathbf{R}$ is common to all three codes and is written as

$$
\dot{\mathbf{R}}-\hat{\mathbf{e}} \cdot \mathbf{R}=\frac{1}{2} \sum_{i}^{4} \frac{\mathbf{r}_{i}}{n_{i}}-\frac{1}{3} \nabla \mu+\mathbf{F},
$$

where $\hat{\mathbf{e}}$ is the velocity gradient tensor and $\mathbf{r}$ is the subchain end-to-end vector. The chemical potential $\mu$ maintains a uniform density with small fluctuations. The corresponding free energy $A$ is written as

$$
A=\left\{\begin{array}{cc}
\varepsilon\left(\frac{\phi(\mathbf{R})}{\langle\phi\rangle}-1\right)^{2}, & \text { for } \phi(\mathbf{R})>\langle\phi\rangle, \\
0, & \text { for } \phi(\mathbf{R}) \leq\langle\phi\rangle,
\end{array}\right.
$$

where $\phi(\mathbf{R})$ is the local node density evaluated in sub-boxes of dimension $1^{3}$, and $\langle\phi\rangle$ is the average value over the system. The third term on the right hand side of Eq. (1) is the random force obeying: $\langle\mathbf{F}\rangle=\mathbf{0}$ and $\left\langle\mathbf{F}(t) \mathbf{F}\left(t^{\prime}\right)\right\rangle=2 \delta\left(t-t^{\prime}\right) \mathbf{I}$. Note that interactions between nodes such as the two-body Lennard-Jones interactions used in MC and MD are not included in this model.

The kinetic equation for monomer transport along the chain is described by a one-dimensional Langevin type kinetic equation containing the same physicochemical forces appearing in Eq. (1), and is also common to all examined codes,

$$
\frac{\dot{n}}{\varphi}=\left(\frac{r_{i}}{n_{i}}-\frac{r_{i-1}}{n_{i-1}}\right)-\frac{1}{3} \nabla \mu+f .
$$

Equation (3) gives the rate of change of $n$ in the strand $i$ due to monomer transport from the neighboring strand $i-1 ; \varphi$ is the local linear density of monomers along the chain given by

$$
\varphi=\frac{1}{2}\left(\frac{n_{i}}{r_{i}}+\frac{n_{i-1}}{r_{i-1}}\right) .
$$

In both Eqs. (3) and (4), $r$ is the magnitude of $\mathbf{r}$. The third term on the right hand side of Eq. (3) is the random force obeying: $\langle f\rangle=0$ and $\left\langle f(t) f\left(t^{\prime}\right)\right\rangle=(2 / 3) \delta\left(t-t^{\prime}\right)$. For numerical convenience, we have introduced a minimum cutoff value for $n$, its normalized value being 0.1 .

In addition to the dynamics described by the equations above, algorithms for the network topological rearrangement, i.e., for creation and destruction of the sliplinks are 


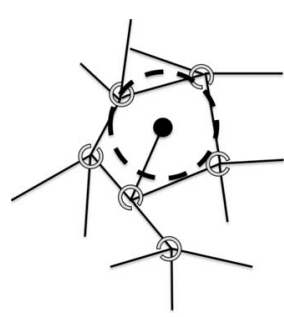

(a)

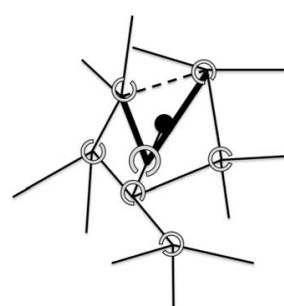

(b)

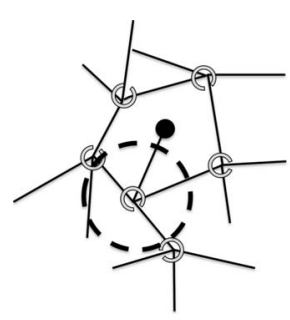

(a)

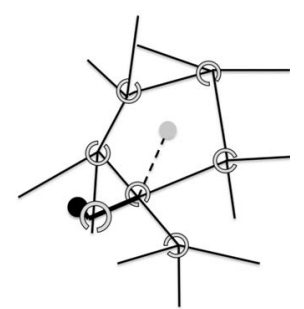

(b)
FIG. 1. Schematic of the sliplink creation process for the original NR scheme: (a) scanning for a partner subchain, with chain end and scanning sphere shown as a filled circle and a dashed circle, respectively; (b) creation of the new sliplink, with dashed and bold solid lines indicating the hooked subchain before and after the hooking process, respectively.

required. A common assumption for network rearrangement (NR) is that creation or destruction of a sliplink always involves a chain end. Also common to all codes is that NR is operated with an assigned frequency, i.e., at every time interval $\tau_{\mathrm{NR}}$.

A modification of the NR scheme recently published ${ }^{26}$ has shown some effects on chain statistics. Hence, we will summarize the two schemes earlier reported, and introduce a third possible variant, especially conceived to better account for fluctuations.

\section{B. NR schemes}

\section{The original NR scheme (NR1)}

We have originally introduced a NR scheme which was long used to reproduce the rheology of polymers. ${ }^{19-25}$ In such scheme, the NR event is triggered by the monomer number in the end-subchain, $n_{\text {end }}$. If $n_{\text {end }}$ exceeds a certain maximum, a new sliplink is created. On the contrary, if $n_{\text {end }}$ falls below a minimum, the sliplink next to the end-subchain is removed. The monomer number window triggering the creation or destruction of the sliplink is

$$
0.5<n_{\text {end }}<1.5 \text {. }
$$

Obviously the window extremes are adjustable parameters. The values in Eq. (5) generate reasonable mean properties as shown in the Appendix. The rest of the procedure is as follows. If a creation event is triggered, a new sliplink is created by letting the chain end to "hook" a partner subchain, which is randomly chosen in the surrounding space. Specifically, the candidates to be hooked are all the subchains crossing the sphere that has its center located at the position of the chain end, and radius equal to the average segment length $a$ [see Fig. 1(a)]. Once the choice of the partner is made, the new sliplink is located in the middle point of the end segment [see Fig. 1(b)], and monomers are split in equal numbers on the two sides of the new sliplink in each of the two subchains of the hooking process. Note that this scheme involves several parameters, such as location and size of the scanning sphere, position in space of the new entanglement, and monomer number subdivision. Indeed, the following two schemes differ in one or more of these parameters. On the other hand, unhooking details are not discussed here because they do not involve parameters, and are the same in all three schemes.
FIG. 2. Schematic of the sliplink creation process for both the no-stretch and the fluctuating NR schemes.

\section{No-stretch NR scheme (NR2)}

Yaoita et $a{ }^{26}{ }^{26}$ proposed a modified scheme to eliminate some chain stretch observed in the original scheme (see the Appendix). Indeed, scanning of the partner chain is here performed by a sphere which, instead of being located at the very end of the chain, has its center at the sliplink next to the chain end [see Fig. 2(a)]. The radius of the sphere is set to $a$ as in the original scheme. The new sliplink is located at the crossing point between the hooked subchain (randomly chosen) and the scanning sphere [see Fig. 2(b)]. The monomers in the hooked subchain are partitioned so as to achieve the same value of linear density $n / r$ (and hence the same value of the tension) on each side of the new sliplink. The monomers on the end-subchain are partitioned so that the internal subchain is equilibrated, i.e., $r^{2} / n=1$ (implying that the monomer number is $n_{0}$ since the segment length is $a$, the radius of the scanning sphere). For the new end-subchain, the length $r$ is adjusted so as to achieve $r^{2} / n=1$ with $n$ the rest of monomers. Thus also the end-subchain is at equilibrium. Finally, the direction of the new end-subchain is chosen randomly.

\section{Fluctuating NR scheme (NR3)}

It is possible to introduce fluctuations in the NR scheme if we a priori assume some simple distribution functions. In this scheme, we modify the triggering criterion given by Eq. (5) in the previous procedures and explicitly introduce fluctuations in the creation process of sliplinks. The procedure runs as follows. If $n_{\text {end }}>\alpha$ ( $\alpha$ is a parameter), then the possibility of sliplink creation is explored first, else the possibility of sliplink destruction is explored first. Creation occurs if

$$
n_{\mathrm{end}}>-\alpha \ln p,
$$

where $p$ is a random number uniformly distributed in the interval $[0,1]$. (Hence we are sort of assuming that monomers are distributed as $\exp (-n)$ like in the classical theory.) On the contrary, if Eq. (6) is not fulfilled then creation does not occur and destruction is examined. To trigger destruction, we utilize the monomer number $n_{\text {sum }}$, which is $n_{\text {end }}$ plus the monomer number of the subchain next to the end-subchain, and examine the condition described by

$$
n_{\text {sum }}<-\alpha \ln p,
$$

where $p$ is again a random number uniformly distributed in $[0,1]$. If Eq. (7) is fulfilled, then destruction of the sliplink takes place; else destruction does not occur and creation is 
examined (if not examined yet). Consequently, if neither Eq. (6) nor Eq. (7) come out fulfilled, no change in topology takes place. In this work, we chose $\alpha=1.5$ to obtain reasonable mean values (see the Appendix).

When sliplink creation has been accepted, we split $n_{\text {end }}$ in half $\left(\frac{1}{2} n_{\text {end }}\right)$ plus a fluctuation. The fluctuation $\Delta n$ is obtained as

$$
\Delta n= \pm \frac{n_{\mathrm{end}}}{2}(1-\sqrt{p})
$$

where $p$ is a uniformly distributed random number in $[0,1]$, and the plus or minus sign is assigned randomly with $\frac{1}{2}$ probability. Thus $n_{\text {end }}$ is split into $n_{1}=\frac{1}{2} n_{\text {end }}+\Delta n ; n_{2}=\frac{1}{2} n_{\text {end }}-\Delta n$, and $n_{1}$ and $n_{2}$ are assigned to the new subchains, $n_{1}$ being conventionally assigned to the more internal subchain and $n_{2}$ to the new chain end. (Note that in the calculation of $\Delta n$, we have assumed a triangular probability distribution with the maximum at $\frac{1}{2} n_{\text {end }}$, and zero at both ends of the interval, 0 and $n_{\text {end }}$.) If either $n_{1}$ or $n_{2}$ comes out smaller than the cutoff value of 0.1 , then they are changed to 0.1 and $n_{\text {end }}-0.1$. The search for the partner subchain is similar to the previous no-stretch scheme with one important difference. Indeed, as before, the center of the search sphere is located at the last sliplink [see Fig. 2(a)], but the radius $r_{1}$ of the sphere is made to depend on $n_{1}$, and includes a fluctuation as

$$
r_{1}=\sqrt{n_{1}}+\Delta r_{1}
$$

where again the fluctuation $\Delta r_{1}$ is assigned with a triangular probability

$$
\Delta r_{1}= \pm \frac{n_{1}}{2}(1-\sqrt{p})
$$

where $p$ is a random number in the interval $[0,1]$, and the sign is randomly assigned with probability $\frac{1}{2}$. If $r_{1}$ comes out too small and the search sphere does not intersect any other chain, the creation process is rejected. If the intersection is successful, the rest of the procedure (splitting of monomers in the partner subchain) is taken as in the previous no-stretch scheme. In view of the large mobility of chain ends, the value of $r_{2}$ does not seem critical so that we take the mean value as $r_{2}=\sqrt{n_{2}}$ (without fluctuation). The chain end is then located in a random direction at a distance $r_{2}$ from the new sliplink. In the following we will refer to the above three schemes as NR1, NR2, NR3, respectively.

\section{Simulations}

The results reported below were obtained with a simulation cell of size $12^{3}$ and a node density $[\langle\phi\rangle$ in Eq. (2)] of 5 (i.e., subchain density is approximately 10). Monodisperse linear polymers were considered with a total number of normalized monomers per chain (actually subchains per chain), $n_{\text {tot }}$, ranging from 5 to 40 . The parameter $\varepsilon$ in Eq. (2) was fixed at 0.5 for NR1 and at 4.0 for NR2 and NR3. These $\varepsilon$ values were chosen large enough to prevent network nodes from clustering, and small enough to avoid artificial effects on dynamics. The frequency of NR events, i.e., the time interval $\tau_{\mathrm{NR}}$, is another parameter, typically fixed at $\tau_{\mathrm{NR}}=\tau$. We have explored different values for $\tau_{\mathrm{NR}}$ but no significant

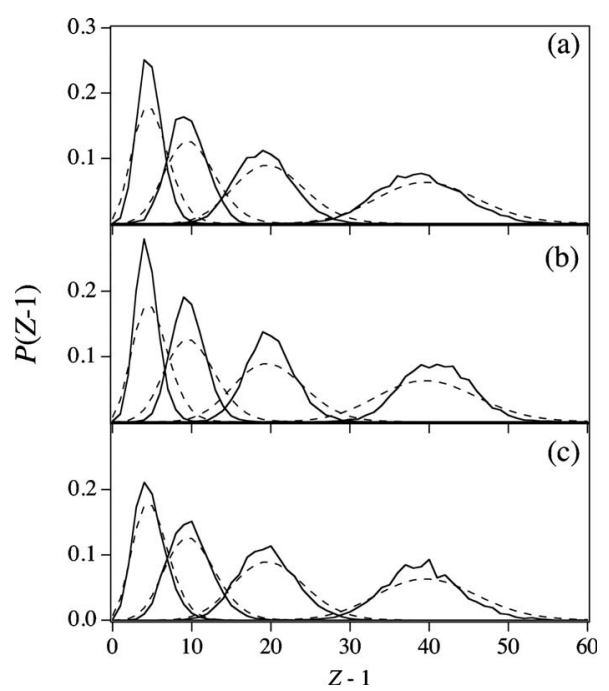

FIG. 3. Distribution of the sliplink number per chain, $Z-1$, for NR1, NR2, and NR3 schemes from top to bottom. Four values of $n_{\text {tot }}$ are shown, namely 5, 10, 20, and 40. The corresponding Poisson distributions are indicated by dashed lines.

changes in the distributions to be discussed next were observed. The distribution functions were obtained from ten independent simulation snapshots under equilibrium conditions. Thus the distributions for subchain length and monomer number were obtained from more than 170000 subchains. The distribution of subchain number per chain is defined over chains (not subchains), and thus the ensemble is smaller. For instance, for the longest chain consisting of $n_{\text {tot }}=40$, the distribution is obtained from approximately 4300 chains.

\section{RESULTS AND DISCUSSION}

Figure 3 shows distributions of the number $Z-1$ of sliplinks per chain ( $Z$ being the number of subchains). Each panel refers to one of the NR schemes previously described. Within all of the panels, several chain lengths are considered, namely $n_{\text {tot }}=5,10,20$, and 40 . Poisson distributions for $\langle Z\rangle$ equal to 5, 10, 20 and 40, respectively, are also shown for comparison. (As shown in the Appendix, $\langle Z\rangle$ and $n_{\text {tot }}$ essentially coincide.) Indeed Poisson distributions were predicted by Schieber. ${ }^{16}$ Although the distributions obtained from simulations with the various NR schemes are not very different from one another, and generally narrower than the corresponding Poissonians, those obtained with the NR3 scheme are slightly closer. The existing microscopic simulations give different results in this respect. Indeed, while the atomistic simulations of Foteinopoulou et al. ${ }^{6}$ show excellent agreement, both the on-lattice simulations of Shanbhag and Larson $^{8}$ and the other atomistic simulations of Tzoumanekas and Theodorou ${ }^{7}$ indicate that deviations exist, with the simulated distributions somewhat narrower (and perhaps more skewed) than the Poissonians. Gaussian distributions could fit our simulations better than Poissonian ones since they have two adjustable parameters instead of just one. In any event, as mentioned in the Sec. I, a Gaussian distribution is asymptotically approached with increasing $\langle Z\rangle .^{7}$ In conclu- 


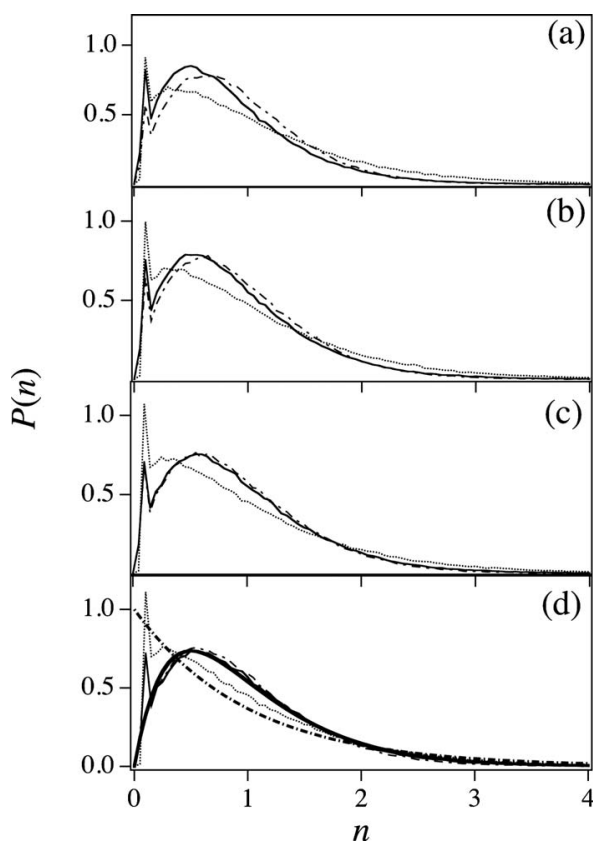

FIG. 4. Distribution of the subchain monomer number, $n$, for $n_{\text {tot }}=5,10,20$, and 40, from top to bottom. Dotted, dot-dashed, and solid lines refer to NR1, NR2, and NR3 schemes, respectively. In panel (d), the bold dot-dashed line is the single exponential of classical theory, while the bold solid line is Eq (11) with $1 / n_{b}=1.96$ and $1 / n_{c}=2.04$

sion, for what concerns the $Z$ distribution our Brownian simulation results are comparable to those obtained from less coarse-grained models.

Figure 4 shows distributions $P(n)$ of the number $n$ of monomers in subchains for various chain lengths $n_{\text {tot }}$. Comparison between panels clearly shows that $P(n)$ soon reaches independence from chain length with growing $n_{\text {tot }}$. In each panel, the three NR schemes are compared. According to the classical theory, ${ }^{13,14}$ the distribution should be a single exponential function [shown in Fig. 4(d) as a bold dot-dashed line], but the results show a clear maximum around $n=0.5$. (Note that the spike at $n=0.1$ is an artifact due to the cutoff needed for numerical reasons, and can be disregarded.) The exact position of the maximum varies with the NR scheme, especially for short chains where results are more sensitive to the hooking rules. It is noted, however, that the distributions resulting from the NR1 scheme remain separated from those of the other two even for long chains, i.e., they stay closer to the exponential distribution. This might perhaps be explained as an effect of the stretch induced by the hooking event in the NR1 scheme. The stretch in the chain ends draws monomers from the internal subchains, which therefore become monomer depleted. Concerning the presence of a maximum (as opposed to the monotonic single exponential), possible explanations were proposed in the literature. Specifically, as mentioned in the Sec. I, Tzoumanekas and Theodorou ${ }^{7}$ introduce the "blocking event" idea whereby, moving along a chain, once an entanglement is found, there exists a chain segment adjacent to it where the occurrence of another entanglement is prohibited. Assuming that the blocking event and the creation of another entanglement both generate single exponential distributions of the corresponding lengths (in monomer spacing), the overall $n$ distribution function comes out as

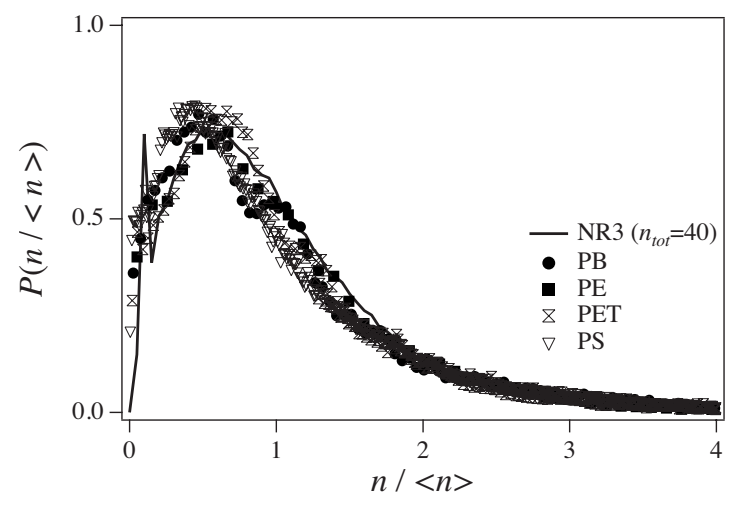

FIG. 5. Comparison of the monomer distribution predicted by our simulation (with NR3) to data (symbols) from Refs 7, 10, and 11.

$$
P(n)=\frac{1}{n_{b}-n_{c}}\left[\exp \left(-\frac{n}{n_{b}}\right)-\exp \left(-\frac{n}{n_{c}}\right)\right]
$$

where $n_{b}$ and $n_{c}$ are average monomer numbers for the blocking and creation events. The prediction of Eq. (11) is shown as a bold line in Fig. 4(d) (parameters being $1 / n_{b}$ $=1.96$ and $1 / n_{c}=2.04$ ) that gives a good agreement with our results obtained with the NR2 and NR3 schemes. Note that the MC results by Tzoumanekas and Theodorou ${ }^{7}$ are reproduced by using $1 / n_{b}=1.30$ and $1 / n_{c}=3.78$, which give a slightly smaller $n$ value for the peak position. Indeed, the curve is not particularly sensitive to the parameter values. For the observed maximum in the $n$ distribution, Greco ${ }^{18}$ offers a different explanation that, in view of the smallness of $\langle n\rangle$, is based on the "thermodynamics of small systems." The distribution function comes out as

$$
\begin{aligned}
P(n) \propto & s(m) \sqrt{n} \exp \left(-m^{2} n\right)+\sqrt{\pi}\left(\frac{1}{2}+s^{2}(m) n\right) \\
& \times[1+\operatorname{erf}(s(m) n)] \exp \left[\left(\frac{1}{m^{2}}-2\right) n\right],
\end{aligned}
$$

where $s(m)=\left(m^{2}-1\right) / m$ and $m$ is an effective chemical potential for monomer transport across entanglements in the sliding diffusive motion of the chain. Using the best-fit value of $m$, the prediction of Eq. (12) almost coincides with the prediction of Eq. (11), and is therefore not shown in Fig. 4.

Theories apart, Fig. 5 directly compares our simulation results obtained with the NR3 scheme (for $n_{\text {tot }}=40$, although the value of $n_{\text {tot }}$ is essentially irrelevant) with the atomistic simulations for PB1000 and PE1000 (Ref. 7) (corresponding to $\langle Z\rangle \approx 12$ and $\langle Z\rangle \approx 34$, respectively), and with the more coarse-grained ones for PET (Ref. 10$)(\langle Z\rangle \approx 7)$ and PS (Ref. 11) $(\langle Z\rangle \approx 16)$. Now, although polystyrene (PS) and polyethyleneterephthalate (PET) results seem to show some deviations, as mentioned already by Spyriouni et al. ${ }^{11}$ all data in Fig. 5 essentially superimpose, and also our Brownian simulations fall reasonably in line in spite of the difference in treating interbead interactions.

Figure 6 shows distributions $P(r)$ of the subchain length, $r$. It is noted that $P(r)$ is insensitive to $\langle Z\rangle$ even more than $P(n)$ and is also indifferent to the NR scheme adopted. The bottom panel in Fig. 6 shows comparison with the Schieber ${ }^{16}$ and $\mathrm{Greco}^{18}$ prediction, i.e., with the expression 


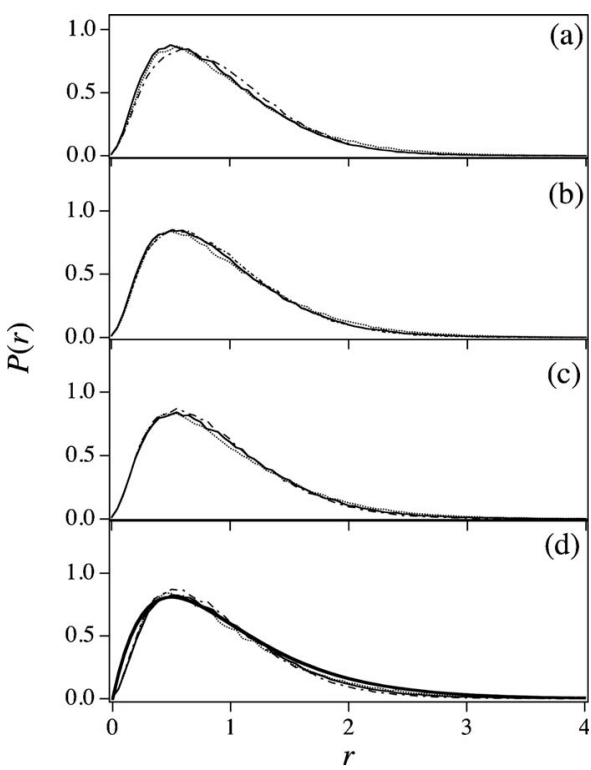

FIG. 6. Distribution of the subchain length, $r$, for $n_{\text {tot }}=5,10,20$, and 40 , from top to bottom. Dotted, dot-dashed and solid lines refer to the NR1, NR2, and NR3 schemes, respectively. Bold line in panel (d) is Eq. (13) with $\langle r\rangle=1$.

$$
P(r) \propto r \exp \left(-2 \frac{r}{\langle r\rangle}\right) .
$$

Indeed, as noted by Greco, ${ }^{18}$ Eq. (13) remains the same both in classical and in small-system thermodynamics. Simulations appear in good agreement with Eq. (13) (with $\langle r\rangle=1$ ).

Figure 7 directly compares our simulations with those obtained by less coarse grained models, specifically by the lattice MC model of Shanbhag and Larson, ${ }^{8}$ the MC advanced model of Foteinopoulou et al., ${ }^{6}$ and the atomistic approach of Tzoumanekas and Theodorou. ${ }^{7}$ (Notice that $P(r)$ was not reported in Ref. 7 and the unpublished data here shown in Fig. 7 were kindly provided by Dr. Tzoumanekas.) Reasonable agreement is observed among all models including our Brownian simulations, in spite of the difference between lattice space $^{8}$ and real space, ${ }^{6,7}$ as well as in interbead interactions.

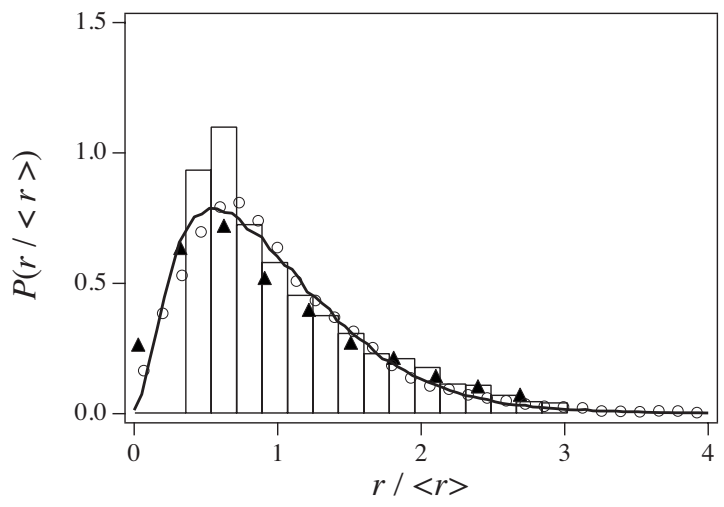

FIG. 7. Comparison to data of the subchain length distribution predicted by our simulation. Triangles are data for C1000 from Fig. 15 of Ref. 6; circles are data for PB1000 (Ref. 7); the histogram is for $\mathrm{N}=500$ from Fig. 3 of Ref. 8.

\section{CONCLUSIONS}

Using our model for the Brownian dynamics of entangled polymer networks we have derived, in the absence of flow, the distribution functions of the relevant single chain properties, namely the distribution $P(Z)$ of the number of entanglements per chain, the monomer distribution $P(n)$ in subchains, and the subchain length distribution $P(r)$. In obtaining these results, we have adopted three different schemes for the constraint renewal, i.e., for the hookingunhooking processes of chain ends. Some of the results obtained are not sensitive to the specific choice, and in general all three schemes give the same qualitative predictions. Whenever quantitative differences are found, the scheme that best compares with the existing evidence in the literature appears to be NR3, although the simpler NR2 is most often entirely equivalent.

Comparison of our predictions with those obtained by lattice and atomistic simulations ${ }^{6-8,10,11}$ shows the following. (i) Differently from Ref. 6, our $Z$ distributions are not strictly Poissonian, similarly to the results obtained in Refs. 7 and 8 (in Ref. 8 only one out of three reported distributions seems Poissonian). (ii) The distribution of monomers in subchains, $P(n)$, is in reasonable agreement with the results in Refs. 7, 10 , and 11, there interpreted in terms of "blocking events;" $P(n)$ is not considered in Ref. 8, and the $P(n)$ reported in Ref. 6 is not determined directly, but only through $P(Z)$. (iii) The distribution of subchain length, $P(r)$, is also in reasonable agreement with similar distributions reported in Refs. 6-8. All in all, therefore, we may conclude that, at least for these observables, our Brownian simulations realize networks the properties of which are consistent with those for the shortest path networks that are deduced by a coarsegraining bottom-up approach, starting from lattice or atomistic models.

Comparison with the existing theories confirms that the monomer distribution $P(n)$ is not a single exponential and shows the maximum predicted by Greco ${ }^{18}$ and by the "blocking" idea of Tzoumanekas and Theodorou. ${ }^{7}$ The subchain length distribution, $P(r)$, is well described by Eq. (13), independently predicted by Schieber ${ }^{16}$ and Greco. ${ }^{18}$

Two further comments before concluding. In all three NR schemes here explored, we have not explicitly checked that hooking/unhooking processes fulfill detailed balance, nor would it be easy to perform such a check. Results, however, do not show any pathological behavior. Second, equilibrium distribution functions might be derivable intrinsically from the Langevin-type kinetic equations for chain dynamics. Some of us are working along this direction and results will be reported in the future.

\section{ACKNOWLEDGMENTS}

The authors thank Dr. C. Tzoumanekas of the National Technical University of Athens for his courtesy in providing us with data reported in Figs. 5 and 7.

\section{APPENDIX: BASIC TESTS FOR NR SCHEMES}

We here report some mean values of relevant equilibrium properties, the scaling behavior of some dynamical 


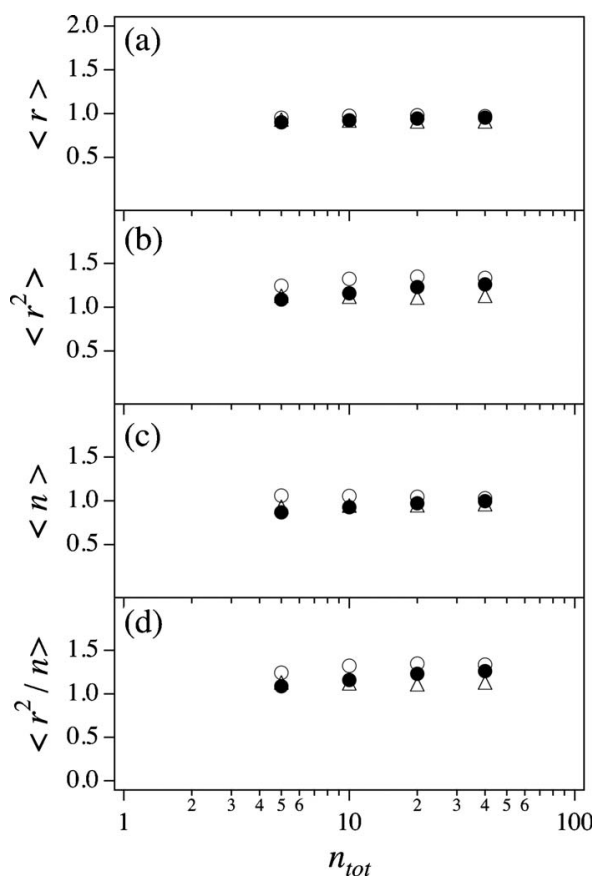

FIG. 8. Average values of subchain properties vs molecular weight. Empty circles and triangles, and filled circles refer to NR1, NR2, and NR3, respectively.

properties, and the linear viscoelastic response, as obtained from the three NR schemes examined in the text. Figure 8 shows mean values of subchain length, square subchain length, monomer number, and square subchain length divided by the monomer number, the latter quantity representing the trace of the stress tensor, and hence a measure of chain stretch. These subchain properties look reasonable for all schemes, except perhaps for the original NR1 scheme that

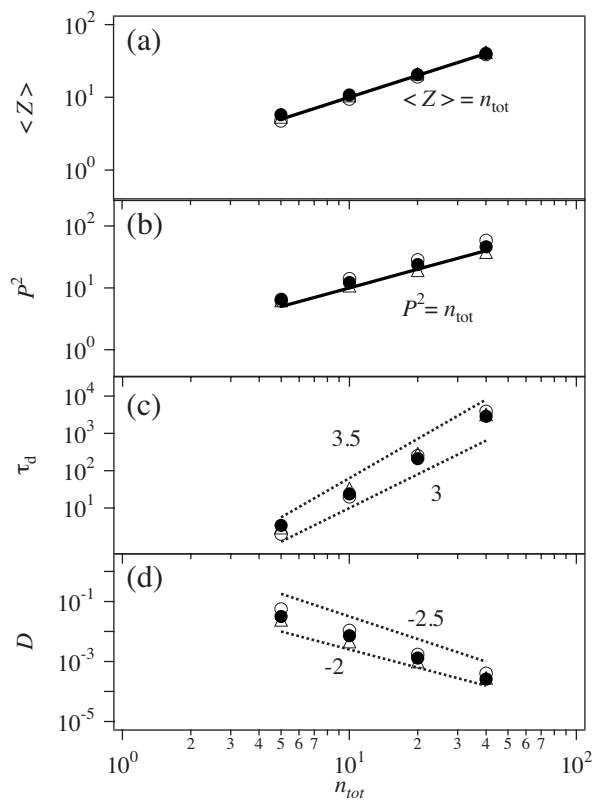

FIG. 9. Scaling of several chain and network properties with molecular weight: (a) average subchain number per chain, (b) square end-to-end distance, (c) longest relaxation time, (d) diffusion coefficient. Solid lines indicate equality, while dotted lines show typical scaling exponents. Empty circles and triangles, and filled circles refer to NR1, NR2, and NR3, respectively.

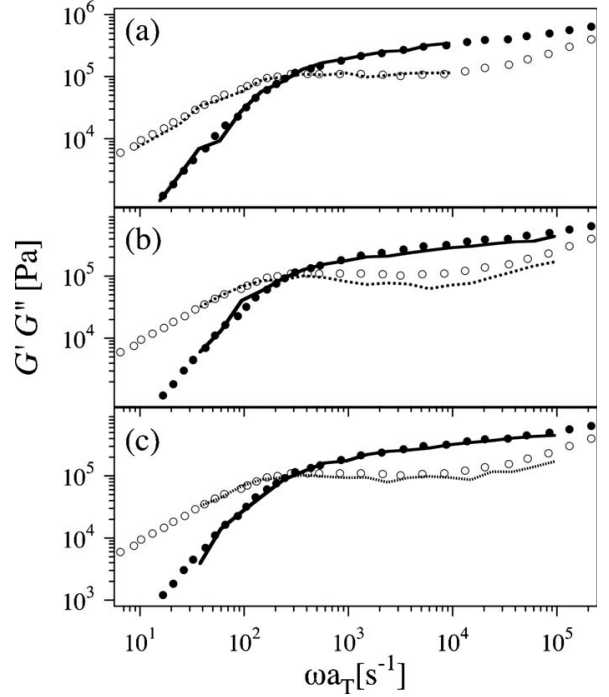

FIG. 10. Viscoelastic moduli $G^{\prime}$ (solid line) and $G^{\prime \prime}$ (dotted line) from the NR1, NR2, and NR3 schemes, from top to bottom. Symbols are experimental data for monodisperse linear polyisoprene from Ref. 27. Simulation parameters are in Table I.

shows systematically larger values, owing to some chain stretch induced by the hooking rule described in Fig. 1 of the text. Figure 9 shows the molecular weight scaling of static and dynamic chain properties. In particular, the top panel reports the average value of subchain number per chain, $\langle Z\rangle$, which is seen to coincide with the (normalized) monomer number $n_{\text {tot. }}$ The other panels in Fig. 9 again indicate reasonable agreement with well established scaling laws for all three NR schemes. (We already reported similar results for NR $1{ }^{19}$ and NR2. ${ }^{26}$ ) Finally, Fig. 10 compares data of linear viscoelasticity moduli for a polyisoprene melt of molecular weight $48.8 \mathrm{k}$ (Ref. 27) with the predictions obtained by our simulations for each of the three schemes. Parameters for these simulations are reported in Table I, where the product of the quantities in the first two columns equals $48.8 \mathrm{k}$. It was already noted ${ }^{26}$ that the subchain molecular weight $M_{0}$ for the no-stretch NR scheme comes out significantly smaller than that for the original NR scheme, and that for both schemes $M_{0}$ is significantly smaller than the classical (Ferry) value for the entanglement molecular weight $M_{\mathrm{e}},{ }^{28}$ possibly due to the effect of node fluctuations. ${ }^{20}$ In Table I, the basic modulus $G_{0}$ was calculated as $G_{0}=\rho R T / M_{0}$, where $\rho$ is density, whereas the characteristic time $\tau$ is determined from the horizontal shift needed to fit $G^{\prime}$ and $G^{\prime \prime}$ data. Reflecting the difference in $M_{0}$, for the original scheme $\tau$ is larger than for the no-stretch scheme. For the fluctuating scheme, all parameters are found to be the same as for the no-stretch scheme. For all three schemes, Fig. 10 shows good, in some case even excellent, agreement with data.

TABLE I. Simulation parameters used for Fig. 10.

\begin{tabular}{lcccc}
\hline \hline Scheme & $\begin{array}{c}M_{0} \\
(\mathrm{kDa})\end{array}$ & $n_{\text {tot }}$ & $\begin{array}{c}G_{0} \\
(\mathrm{~Pa})\end{array}$ & $\begin{array}{c}\tau \\
(\mathrm{s})\end{array}$ \\
\hline Original (NR1) & 3.5 & 14 & $6.3 \times 10^{5}$ & $6.7 \times 10^{-5}$ \\
No-stretch (NR2) & 2.0 & 24 & $1.1 \times 10^{6}$ & $6.7 \times 10^{-6}$ \\
Fluctuating (NR3) & 2.0 & 24 & $1.1 \times 10^{6}$ & $6.7 \times 10^{-6}$ \\
\hline \hline
\end{tabular}


${ }^{1}$ M. Doi and S. F. Edwards, The Theory of Polymer Dynamics (Oxford University Press, New York, 1986).

${ }^{2}$ T. C. B. McLeish, Adv. Phys. 51, 1379 (2002).

${ }^{3}$ R. Everaers, S. K. Sukumaran, G. S. Grest, C. Svaneborg, A. Sivasubramanian, and K. Kremer, Science 303, 823 (2004).

${ }^{4}$ S. K. Sukumaran, G. S. Grest, K. Kremer, and R. Everaers, J. Polym. Sci., Part B: Polym. Phys. 43, 917 (2005).

${ }^{5}$ M. Kroger, Comput. Phys. Commun. 168, 209 (2005).

${ }^{6}$ K. Foteinopoulou, N. C. Karayiannis, V. G. Mavrantzas, and M. Kroger, Macromolecules 39, 4207 (2006)

${ }^{7}$ C. Tzoumanekas and D. N. Theodorou, Macromolecules 39, 4592 (2006).

${ }^{8}$ S. Shanbhag and R. G. Larson, Macromolecules 39, 2413 (2006).

${ }^{9}$ C. Tzoumanekas and D. N. Theodorou, Curr. Opin. Solid State Mater. Sci. 10, 61 (2006).

${ }^{10}$ K. Kamio, K. Moorthi, and D. N. Theodorou, Macromolecules 40, 710 (2007)

${ }^{11}$ T. Spyriouni, C. Tzoumanekas, D. N. Theodorou, F. Muller-Plathe, and G. Milano, Macromolecules 40, 3876 (2007).

${ }^{12}$ S. Shanbhag and M. Kroger, Macromolecules 40, 2897 (2007).

${ }^{13}$ S. F. Edwards and K. E. Evans, J. Chem. Soc., Faraday Trans. 2 77, 1913 (1981).

${ }^{14}$ P. G. de Gennes, J. Phys. (France) 36, 1199 (1975).

${ }^{15}$ E. Helfand and D. S. Pearson, J. Chem. Phys. 79, 2054 (1983).
${ }^{16}$ J. D. Schieber, J. Chem. Phys. 118, 5162 (2003).

${ }^{17}$ J. D. Schieber, J. Neergaard, and S. Gupta, J. Rheol. 47, 213 (2003).

${ }^{18}$ F. Greco, Eur. Phys. J. E 25, 175 (2008).

${ }^{19}$ Y. Masubuchi, J. I. Takimoto, K. Koyama, G. Ianniruberto, G. Marrucci, and F. Greco, J. Chem. Phys. 115, 4387 (2001).

${ }^{20}$ Y. Masubuchi, G. Ianniruberto, F. Greco, and G. Marrucci, J. Chem. Phys. 119, 6925 (2003).

${ }^{21}$ Y. Masubuchi, G. Ianniruberto, F. Greco, and G. Marrucci, Modell. Simul. Mater. Sci. Eng. 12, S91 (2004).

${ }^{22}$ Y. Masubuchi, G. Lanniruberto, F. Greco, and G. Marrucci, J. NonNewtonian Fluid Mech. 149, 87 (2008).

${ }^{23}$ Y. Masubuchi, H. Watanabe, G. Ianniruberto, F. Greco, and G. Marrucci, Macromolecules 41, 8275 (2008).

${ }^{24}$ K. Furuichi, C. Nonomura, Y. Masubuchi, H. Watanabe, G. Ianniruberto, F. Greco, and G. Marrucci, Rheol. Acta 47, 591 (2008).

${ }^{25}$ Y. Masubuchi, K. Furuichi, K. Horio, T. Uneyama, H. Watanabe, G. Ianniruberto, F. Greco, and G. Marrucci, J. Chem. Phys. 131, 114906 (2009).

${ }^{26}$ T. Yaoita, T. Isaki, Y. Masubuchi, H. Watanabe, G. Ianniruberto, F. Greco, and G. Marrucci, J. Chem. Phys. 128, 154901 (2008).

${ }^{27}$ Y. Matsumiya, H. Watanabe, and K. Osaki, Macromolecules 33, 499 (2000).

${ }^{28}$ R. G. Larson, T. Sridhar, L. G. Leal, G. H. McKinley, A. E. Likhtman, and T. C. B. McLeish, J. Rheol. 47, 809 (2003). 